\title{
A Comparison of Salivary Mercury Levels in Children with Attention Deficit/Hyperactivity Disorder When Compared to Age-matched Controls: A Case-control Observational Study
}

\author{
Mohammed J Barry ${ }^{1}$, Fatmah Almotawah ${ }^{2}$, Sharat C Pani ${ }^{3}$, Navin A Ingle ${ }^{4}$
}

\begin{abstract}
Aim: The aim of this study was to compare the level of mercury in the saliva of children with attention deficit/hyperactivity disorder (ADHD) as compared to age- and gender-matched controls in specific age groups.

Materials and methods: A case-control observational study design was used. In school children with ADHD and outpatient dental clinics of a university dental hospital, the participants were schoolchildren diagnosed with ADHD studying in the first grade (6-7 years), sixth grade (12-13 years), and ninth grade (15-16 years) and were gender-matched to children without ADHD attending regular classes in school. Ninety children with ADHD comprised the test group while 90 children without ADHD comprised the control group.

Results: In this study, we found that children with ADHD had higher levels of salivary mercury than their age- and gender-matched counterparts; however, this difference was significant only in the 6-7 years of age group. The regression model showed a mild positive association between salivary mercury and ADHD; however, the association was not statistically significant.

Conclusion: While there is some indication that salivary mercury may be higher in children with ADHD, there is insufficient evidence to establish a definite association between the two.

Clinical significance: The study highlights the need to evaluate existing evidence on the role of mercury, especially salivary mercury, in ADHD. Keywords: Attention deficit hyperactivity disorder, Dental amalgam, Mercury, Saliva.

The Journal of Contemporary Dental Practice (2020): 10.5005/jp-journals-10024-2747
\end{abstract}

\section{INTRODUCTION}

Attention deficit/hyperactivity disorder (ADHD) is one of the most common mental disorders affecting children. Attention deficit/ hyperactivity disorder also affects many adults. Symptoms of ADHD include inattention (not being able to keep focus), hyperactivity (excess movement that is not fitting to the setting), and impulsivity (hasty acts that occur in the moment without thought). ${ }^{1}$

The prevalence of ADHD in children in the west has been reported as being between 2\% and 5.5\% ${ }^{1,2}$ In Saudi Arabia, there have been studies that have placed the prevalence of ADHD at being between $2.7 \%$ and $4.2 \%$ on average, ${ }^{3}$ with reports of incidence as high as $7 \%$ in male students. ${ }^{3-5}$ It is generally accepted that the prevalence of ADHD reduces with age with the typical adult rates being between $1.2 \%$ and $2.7 \%{ }^{2}$

The role of heavy metals in the development of the brain and neurological disorders is well-documented..$^{6-8}$ Of the several heavy metals, mercury has received the greatest attention as it is associated with both seafood as well as several preservatives used in food ingredients. ${ }^{6,9-11}$ However, the role that mercury has played in the incidence of diseases such as Autism or ADHD has been controversial in the past. There have been several studies to show that the trace amounts of mercury found in vaccines or in dental products may be associated with ADHD; however, the models proposed fail to prove a causal relationship. ${ }^{12-14}$

Saliva is a useful noninvasive screening tool for several heavy metals, including mercury. Salivary analysis of mercury provides a relatively easy measure to compare mercury levels in children with ADHD and those without ADHD. ${ }^{15-18}$ While few studies have successfully shown increased mercury levels in the blood, urine, and

\footnotetext{
1,2Department of Preventive Dental Sciences, Riyadh Elm University, Riyadh, Kingdom of Saudi Arabia

${ }^{3}$ Department of Paediatric Dentistry, Schulich School of Medicine and Dentistry, Western University, London, Ontario, Canada

${ }^{4}$ Department of Dental Public Health, Riyadh Elm University, Riyadh, Kingdom of Saudi Arabia
}

Corresponding Author: Mohammed J Barry, Department of Preventive Dental Sciences, Riyadh Elm University, Riyadh, Kingdom of Saudi Arabia, Phone: +966 543222108, e-mail: mohammed.j.barry@ student.riyadh.edu.sa

How to cite this article: Barry MJ, Almotawah F, Pani SC, et al. A Comparison of Salivary Mercury Levels in Children with Attention Deficit/Hyperactivity Disorder When Compared to Age-matched Controls: A Case-control Observational Study. J Contemp Dent Pract 2020;21(2):129-132.

Source of support: Nil

Conflict of interest: None

saliva of individuals with $A D H D,{ }^{13,14,19}$ there has been no systematic attempt made to evaluate the age, demographic, or oral health factors that could influence the levels of mercury in the saliva of children with ADHD when compared to age- and gender-matched controls.

The study of oral health of children is difficult to calibrate due to the changing dentition and the corresponding oral health changes associated with them. The World Health Organization (WHO) has therefore suggested the use of specific ages or age groups to make sample collection easier. They recommend the age of 5 years to evaluate children in the primary dentition, 12 years to record the 
effect of mixed dentition, and 15 years to record the initial effects of young permanent dentition. ${ }^{20}$

The aim of this study was to compare the level of mercury in the salvia of children with ADHD as compared to age- and gendermatched controls in specific age groups. The study also sought to examine the role of maternal salivary mercury levels, if any, on the presence or absence of ADHD in the children.

\section{Materials and Methods}

\section{Study Design}

A case-control, observational study design was used.

\section{Ethical Approval}

The proposal was registered with the research center of the Riyadh Elm University and Ethical Approval was obtained from the Institutional Review Board (IRB) of the Riyadh Elm University (RC/ IRB/2019/64). Approval was obtained from the Al Jawdah Private school in Riyadh city for children with ADHD for examining the medical records of the children. Informed consent was obtained from the parents of all children before accessing records and examination. Verbal assent of the children was obtained before examination and collection of saliva.

\section{Sample Power Calculation}

The sample power was calculated using a sample power calculator (G-Power 3.0, Universtat Kiel, Germany). It was estimated that in order to obtain an effect size of 0.5 and a power of 0.95 , a minimum of 30 participants per group were required. Using the presence or absence of ADHD and the three index age groups as indicators, it was determined that the sample would need 180 participants to achieve the required power.

\section{Sample Setting and Selection}

The sample was drawn from schoolchildren studying in the first grade (6-7 years), sixth grade (12-13 years), and ninth grade (15-16 years). Children diagnosed with ADHD as per the Vanderbilt diagnostic score and attending a special school for children with ADHD comprised the test group. The sample comprised of 30 children with ADHD and 30 controls in each age group. Ninety children with ADHD comprised the test group while 90 children without ADHD comprised the control group. These children were age- and gender-matched to children without ADHD attending regular classes in school. Recruitment of the sample was stopped once the required sample size had been reached.

\section{Patient and Public Involvement}

Patients were involved in this study at the stage of data collection. The parents of the patients were informed about the overall aim of the study. Public involvement in the recruitment stage of the study was limited to the involvement of the coordinators of the special schools.

\section{Variables Recorded}

Saliva was collected from children using the passive drool technique and stored in an icebox at $\sim 5^{\circ}$ and transported to a deep freezer where the collected samples were stored at $-60^{\circ} \mathrm{C}$ until the time of analysis. Salivary mercury levels were measured using atomic absorption spectroscopy. The children were examined for the presence of silver amalgam restorations. Children with dental amalgam restorations were excluded from the study in order to avoid the confounding effect of mercury from the amalgam restorations.

\section{Mercury (Hg) Determination}

Cold vapor atomic absorption spectrometry (CV-AAS) (PerkinElmer FIMS 400-Flow Injection Mercury System) was used to determine the total $\mathrm{Hg}$. Saliva samples $(1 \mathrm{~mL})$ were digested with $3 \mathrm{~mL}$ of concentrated nitric acid (Merck Suprapur, Merck KgaA, Frankfurter Strasse 250, Darmstadt) in Teflon vessels under a pressure at $120^{\circ} \mathrm{C}$ for 8 hours. ${ }^{21,22}$ Each series of analyses was accompanied by concurrent mineralization and identification of Standard Reference Materials (SRMs) no. 1577b "Bovine Liver" from NIST Gaithersburg, USA). Batches with accompanying SRMs outside the certified range were repeated. For quantitation, a mercury standard for atomic absorption from $\mathrm{C}$. Erba reagents $1(\mathrm{mg} / \mathrm{mL})$ was used and the detection limit was $0.1(\mathrm{ng} / \mathrm{mL})$. The reliability of $\mathrm{Hg}$ determination, expressed as the coefficient of variation on repeated assays of the same samples, was below $5 \%$.

\section{Data Analysis}

Salivary mercury levels were tested using the tests for kurtosis and skew and were found to be normally distributed. The data were then analyzed using parametric statistics. The independent samples $t$ test was used to compare the mercury levels between children with ADHD and those without in each age group. A logistic regression model with the presence or absence of ADHD as the dependent variable was generated to explore the effects of the different recorded variables on ADHD.

\section{Results}

The sample was comprised of a total of 180 children ( 86 females and 94 males) equally distributed $(n=60)$ into the index age groups of 6-7 years ( 32 females and 28 males), 12-13 years ( 34 females and 26 males), and $15-16$ years ( 20 females and 40 males). Each index age group comprised of a case group of 30 children with ADHD who were age- and gender-matched with a control group.

When the salivary mercury levels were compared between males and females, it was observed that although females had a slightly higher mean salivary mercury level $(5.87 \mu \mathrm{g} / \mathrm{L}, \mathrm{SD} \pm 1.2 \mu \mathrm{g} / \mathrm{L})$ than males $(5.75 \mu \mathrm{g} / \mathrm{L}, \mathrm{SD} \pm 1.4 \mu \mathrm{g} / \mathrm{L})$, these differences were not statistically significant $(t=-532, p=0.561)$. When the salivary mercury levels were compared among the different age groups, it was observed that the 6-7-year-old age group had a significantly lower salivary mercury level than the other two age groups (Table 1).

Children with ADHD had higher salivary mercury levels than children without ADHD. However, these differences were only significant in the 6-7-year-old age group (Table 2).

The binary logistic regression model showed that although there was a positive association between salivary mercury levels of the children and ADHD, the strength of association fell short of being statistically significant ( $p=0.67$ ) (Table 3 ).

\section{Discussion}

There is an increased concern over the role of mercury in the etiology of neurodevelopmental disorders of childhood especially ADHD. ${ }^{1-3}$ Despite the known detrimental effect of mercury on the development of the central nervous system, definitive proof of association has remained elusive. ${ }^{4,5}$ The aim of this study was to examine the levels of salivary mercury in children with ADHD and compare them to age- and gender-matched controls. 
Factors Influencing Salivary Mercury Level

Table 1: Comparison of salivary mercury levels among age groups

\begin{tabular}{|c|c|c|c|c|c|c|}
\hline & \multirow[b]{2}{*}{ Mean } & \multirow[b]{2}{*}{ Standard deviation } & \multicolumn{2}{|c|}{ 95\% confidence interval for mean } & \multirow[b]{2}{*}{$F^{a}$} & \multirow[b]{2}{*}{ Sig } \\
\hline & & & Lower bound & Upper bound & & \\
\hline $6-7$ years $^{c}$ & 5.4967 & 1.31187 & 5.1578 & 5.8356 & & \\
\hline $12-13$ years $^{d}$ & 6.1233 & 1.45734 & 5.7469 & 6.4998 & 3.561 & $0.030^{\mathrm{b}}$ \\
\hline $15-16$ years $^{d}$ & 5.8250 & 1.05888 & 5.5515 & 6.0985 & & \\
\hline Total & 5.8150 & 1.30489 & 5.6231 & 6.0069 & & \\
\hline
\end{tabular}

${ }^{\mathrm{a}}$ Significance calculated using the one-way ANOVA

${ }^{\text {bDifferences significant at } p<0.05}$

${ }^{c, \mathrm{~d} D}$ Differences in superscript denote difference significant at $p<0.05$ when compared using the Tukey's highest significant difference (HSD) test

Table 2: Comparison of mean salivary mercury levels (in $\mu \mathrm{g} / \mathrm{L}$ ) between children with attention deficit/hyperactivity disorder (ADHD) and those without ADHD

\begin{tabular}{llllll}
\hline Age & ADHD & Mean & Standard Deviation & $t^{a}$ & 5.453 \\
6-7 years & ADHD & 6.58 & 0.94 & 0.43 & 0.682 \\
$12-13$ years & No ADHD & 4.41 & $0.691^{\mathrm{b}}$ & 0.323 \\
& ADHD & 6.32 & 0.98 & 0.383 & 0.732 \\
\hline
\end{tabular}

${ }^{\mathrm{a}}$ Calculated using the student's $t$ test

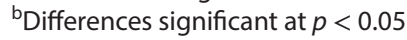

Table 3: Binary logistic regression model with presence or absence of ADHD as the dependent variable

\begin{tabular}{llllll}
\hline Variables in the equation $^{a}$ & \multicolumn{1}{l}{} \\
\hline & $B$ & S.E. & Wald & $d f$ & Significance \\
\hline Age group & -0.149 & 0.215 & 0.480 & 1 & 0.488 \\
Salivary mercury child & -0.150 & 0.131 & 1.308 & 1 & 0.253 \\
Salivary mercury mother & 0.083 & 0.177 & 0.219 & 1 & 0.640 \\
Constant & 0.926 & 2.139 & 0.188 & 1 & 0.662 \\
\hline
\end{tabular}

${ }^{a}$ Variable(s) entered on step 1: maternal amalgam, age group, salivary mercury (child), salivary mercury (adult)

While blood mercury testing remains the definitive source for the testing of mercury levels, there have been studies that have focused on the use of noninvasive samples such as saliva and hair to determine mercury levels. ${ }^{4-7}$ The development of CV-AAS for the determination of mercury levels has been shown to be an effective measure of mercury in both food and substrates such as saliva. ${ }^{5}$ Given the age of the population being studied and the accuracy of measures such as CV-AAS, we decided to use saliva as the sample for mercury in children.

The fact that we found mildly elevated levels of mercury in the salvia of children with ADHD is not conclusive of any causative relationship. However, when combined with the mildly positive association seen in the children, it seems to suggest merit to the theory proposed by some authors that mercury may play an associative rather than causative role in the development of ADHD. ${ }^{8-11}$

The use of mercury containing dental restorations such as silver amalgam fillings in mothers at the time of pregnancy has been a controversial topic. ${ }^{9}$ The results of our matched study design seem to suggest that when controlled for age and gender, we were unable to establish a significant association between the presence of a mercury-containing dental restoration in the mother's oral cavity at the time of pregnancy and the presence of ADHD. A recent study by Lin et al. conducted in a sample of 80,000 mothers in the United States showed that although there was a weak positive association between the presence of amalgam restorations and ADHD, there was insufficient evidence to establish any meaningful relationship. ${ }^{19}$ The results of our study seem to support this hypothesis and are in keeping with the studies of Karouna-Renier et al. ${ }^{9}$ and Van Wijngaarden et al. ${ }^{11}$ that suggest a low level of evidence between prenatal mercury level and ADHD.

One of the limitations of using saliva as a sample to estimate mercury is the possible confounding factors that dentition, diet, and development play in the expression of salivary metals. However, to overcome this bias, the WHO suggests the use of index age groups. ${ }^{12}$ The index age groups used in this study suggested that while there was some difference in the salivary mercury levels of children with ADHD and those without in the primary dentition (6-7 years) age group, this difference was not significant with the children in the mixed (12-13 years) and permanent (15-16 years) age groups. ${ }^{12}$ This finding could explain the observations of Lygre et al., who found that the association between the presence of a maternal dental amalgam restoration and ADHD was inversely associated with the age of the child. ${ }^{13}$ While the metabolism of mercury in children is beyond the scope of this study, the results of this study seem to suggest that future research could look into the possible reasons 
for this inverse association between age and mercury levels as it is related to ADHD.

The results of this study should also be viewed keeping in mind the limitations of the observational study design. While all attempts were made to match the age and gender of children being studied, it was not possible to quantify the severity of the ADHD. Given the large variations in the scores used for the diagnosis of ADHD, it was decided to limit the focus of the current study to the presence or absence of ADHD. A further limitation of the current study was that while the presence of maternal dental amalgam restorations was determined clinically, the presence of the restoration during the pregnancy was based on the reported history. Despite these limitations, the fact that there was some positive association between the salivary mercury levels of the child and presence of ADHD seems to suggest that we cannot fully rule out the role mercury in the etiopathogenesis of ADHD.

\section{CONCLUSION}

Within the limitations of the study, we can state that the following:

- Children with ADHD seem to have a significantly higher level of salivary mercury than age- and gender-matched controls; however, this difference is the highest in the primary dentition and reduces in the permanent and mixed dentitions.

- The data in this study do not show sufficient association between ADHD and salivary mercury to determine mercury as a cause of ADHD.

- There seems to be evidence to suggest that the association between salivary mercury and ADHD is not a causative one, but an association that merits further research.

\section{References}

1. Fulton $B D$, Scheffler RM, Hinshaw SP, et al. National variation of ADHD diagnostic prevalence and medication use: health care providers and education policies. Psychiatr Serv 2009;60(8):1075-1083. DOI: 10.1176/ ps.2009.60.8.1075.

2. Simon V, Czobor P, Balint S, et al. Prevalence and correlates of adult attention-deficit hyperactivity disorder: meta-analysis. Br J Psychiatry 2009;194(3):204-211. DOI: 10.1192/bjp.bp.107.048827.

3. Alqahtani MM. Attention-deficit hyperactive disorder in school-aged children in Saudi Arabia. Eur J Pediatr 2010;169(9):1113-1117. DOI: 10.1007/s00431-010-1190-y.

4. Homidi M, Obaidat $Y$, Hamaidi D. Prevalence of attention deficit and hyperactivity disorder among primary school students in Jeddah city, KSA. Life Sci J 2013;10:280-285.

5. Alhraiwil NJ, Ali A, Househ MS, et al. Systematic review of the epidemiology of attention deficit hyperactivity disorder in Arab countries. Neurosciences 2015;20(2):137-144. DOI: 10.17712/ nsj.2015.2.20140678.

6. Bose-O'Reilly S, McCarty KM, Steckling N, et al. Mercury exposure and children's health. Curr Probl Pediatr Adolesc Health Care 2010;40(8):186-215. DOI: 10.1016/j.cppeds.2010.07.002.
7. Azevedo BF, Barros Furieri L, Pecanha FM, et al. Toxic effects of mercury on the cardiovascular and central nervous systems. J Biomed Biotechnol 2012;2012:949048. DOI: 10.1155/2012/949048.

8. Kim S, Arora M, Fernandez C, et al. Lead, mercury, and cadmium exposure and attention deficit hyperactivity disorder in children. Environ Res 2013;126:105-110. DOI: 10.1016/j.envres.2013. 08.008.

9. Karouna-Renier NK, Rao KR, Lanza JJ, et al. Mercury levels and fish consumption practices in women of child-bearing age in the Florida Panhandle. Environ Res 2008;108(3):320-326. DOI: 10.1016/ j.envres.2008.08.005.

10. Geier DA, Hooker BS, Kern JK, et al. A dose-response relationship between organic mercury exposure from thimerosal-containing vaccines and neurodevelopmental disorders. Int J Environ Res Public Health 2014;11(9):9156-9170. DOI: 10.3390/ijerph110909156.

11. Van Wijngaarden E, Thurston SW, Myers GJ, et al. Methyl mercury exposure and neurodevelopmental outcomes in the Seychelles child development study main cohort at age 22 and 24 years. Neurotoxicol Teratol 2017;59:35-42. DOI: 10.1016/j.ntt.2016.10.011.

12. Geier DA, Kern JK, Homme KG, et al. The risk of neurodevelopmental disorders following Thimerosal-containing Hib vaccine in comparison to Thimerosal-free Hib vaccine administered from 1995 to 1999 in the United States. Int J Hyg Environ Health 2018;221(4):677-683. DOI: 10.1016/j.ijheh.2018.03.004.

13. Lygre GB, Aase $\mathrm{H}$, Haug K, et al. Prenatal exposure to dental amalgam and risk of symptoms of attention-deficit and hyperactivity disorder (ADHD). Community Dent Oral Epidemiol 2018;46(5):472-481. DOI: 10.1111/cdoe.12409.

14. Patel NB, Xu Y, McCandless LC, et al. Very low-level prenatal mercury exposure and behaviors in children: the HOME Study. Environ Health. 2019;18(1):4. DOI: 10.1186/s12940-018-0443-5.

15. Zheng $P$, Li M, Jurevic $R$, et al. A gold nanohole array based surfaceenhanced Raman scattering biosensor for detection of silver $(\mathrm{I})$ and mercury(II) in human saliva. Nanoscale 2015;7(25):11005-11012.

16. Wordofa DN, Ramnani P, Tran TT, et al. An oligonucleotidefunctionalized carbon nanotube chemiresistor for sensitive detection of mercury in saliva. Analyst 2016;141(9):2756-2760. DOI: 10.1039/ c6an00018e.

17. Kawde AN. Trace determination of $\mathrm{Hg}$ (II) in human saliva using disposable electrochemically pretreated graphite pencil electrode surfaces. Acta Chim Slov 2017;64(2):267-275. DOI: 10.17344/ acsi.2016.2538.

18. Yilmaz S, Adisen MZ. Ex vivo mercury release from dental amalgam after 7.0-T and 1.5-T MRI. Radiology. 2018;288(3):799-803. DOI: 10.1148/radiol.2018172597.

19. Lin PY, Wang J, Chiang YC, et al. Risk of subsequent attentiondeficit/hyperactivity disorder among children and adolescents with amalgam restorations: a nationwide longitudinal study. Community Dent Oral Epidemiol 2018;46(1):47-53. DOI: 10.1111/cdoe.12327.

20. World Health Organization. Oral health surveys: basic methods. Geneva, Switzerland: World Health Organization; 2013.

21. Chien YC, Feldman CA, Zohn HK, et al. Urinary mercury levels before and after amalgam restoration. Sci Total Environ 1996;188(1):39-47. DOI: 10.1016/0048-9697(96)05155-8.

22. Drexler $\mathrm{H}$, Schaller $\mathrm{KH}$. The mercury concentration in breast milk resulting from amalgam fillings and dietary habits. Environ Res. 1998;77(2):124-129. DOI: 10.1006/enrs.1997.3813. 